\title{
Procalcitonin, mid-regional proadrenomedullin and C-reactive protein in predicting treatment outcome in community-acquired febrile urinary tract infection
}

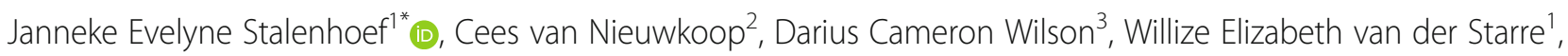
Tanny J. K. van der Reijden ${ }^{1}$, Nathalie Manon Delfos ${ }^{4}$, Eliane Madeleine Sophie Leyten ${ }^{5}$, Ted Koster ${ }^{6}$, Hans Christiaan Ablij', Johannes (Jan) Willem van 't Wout ${ }^{7}$ and Jaap Tamino van Dissel'

\begin{abstract}
Background: A reduction in duration of antibiotic therapy is crucial in minimizing the development of antimicrobial resistance, drug-related side effects and health care costs. The minimal effective duration of antimicrobial therapy for febrile urinary tract infections (fUTI) remains a topic of uncertainty, especially in male patients, those of older age or with comorbidities. Biomarkers have the potential to objectively identify the optimal moment for cessation of therapy.

Methods: A secondary analysis of a randomized placebo-controlled trial among 35 primary care centers and 7 emergency departments of regional hospitals in the Netherlands. Women and men aged $\geq 18$ years with a diagnosis of fUTI were randomly assigned to receive antibiotic treatment for 7 or 14 days. Patients indicated to receive antimicrobial treatment for more than 14 days were excluded from randomization. The biomarkers procalcitonin (PCT), mid-regional proadrenomedullin (MR-proADM), and C-reactive protein (CRP) were compared in their ability to predict clinical cure or failure through the 10-18 day post-treatment visit.

Results: Biomarker concentrations were measured in 249 patients, with a clinical cure rate of 94\% in the 165 randomized and $88 \%$ in the 84 non-randomized patients. PCT, MR-proADM and CRP concentrations did not differ between patients with clinical cure and treatment failure, and did not predict treatment outcome, irrespective of 7 or 14 day treatment duration ( $\operatorname{ROC}_{\mathrm{AUC}} 0.521 ; 0.515 ; 0.512$, respectively). $\mathrm{PCT}$ concentrations at presentation were positively correlated with bacteraemia ( $\tau=0.33, p<0.001)$ and presence of shaking chills $(\tau=0.25, p<0.001)$, and MR-proADM levels with length of hospital stay $(\tau=0.40, p<0.001$ ), bacteraemia ( $\tau=0.33, p<0.001$ ), initial intravenous treatment $(\tau=0.22, p<0.001)$ and time to defervescence $(\tau=0.21, p<0.001)$. CRP did not display any correlation to relevant clinical parameters.
\end{abstract}

Conclusions: Although the biomarkers PCT and MR-proADM were correlated to clinical parameters indicating disease severity, they did not predict treatment outcome in patients with community acquired febrile urinary tract infection who were treated for either 7 or 14 days. CRP had no added value in the management of patients with fUTI.

(Continued on next page)

\footnotetext{
* Correspondence: J.E.Stalenhoef@lumc.nl

'Department of Infectious Diseases, Leiden University Medical Center, POBox

9600, 2300 RC Leiden, the Netherlands

Full list of author information is available at the end of the article
}

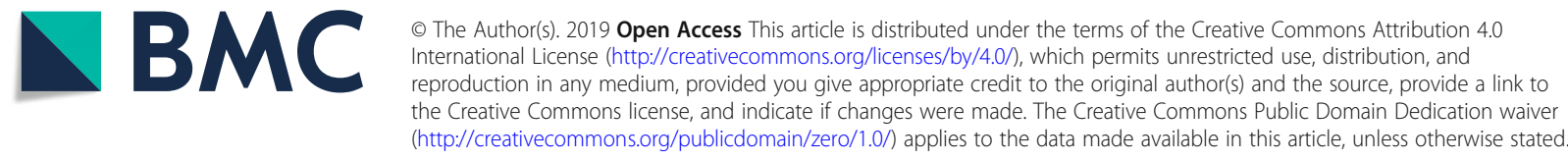


(Continued from previous page)

Trial registration: The study was registered at ClinicalTrials.gov [NCT00809913; December 16, 2008] and trialregister.nl [NTR1583; December 19, 2008].

Keywords: Urinary tract infections, Pyelonephritis, Biomarkers, Treatment duration, Antibiotic therapy, Antibiotic stewardship

\section{Background}

Febrile urinary tract infections (UTI), including acute pyelonephritis and prostatitis, are relatively common in adults, but data on the optimal duration of treatment are limited, especially within men, the elderly and patients with comorbidities. Emerging bacterial resistance calls for more efficient efforts to shorten the duration of antibiotic treatment.

Our previous findings in the FUTIRST trial have shown that patients with community-acquired febrile urinary tract infections, such as women and elderly patients with severe comorbidities, can be safely and efficaciously treated with oral ciprofloxacin for 7 days, irrespective of disease severity upon presentation [1]. In men, however, a short course of therapy can lead to significantly more clinical failures compared to a 14-day course of oral ciprofloxacin.

Previous studies have shown that procalcitonin (PCT) can provide useful guidance for antimicrobial treatment in patients with respiratory tract infections and sepsis [2-4]. However, little is known concerning PCT guided-therapy in patients with urinary tract infections. An earlier subgroup analysis of a randomized trial performed in the intensive care unit (ICU) has shown that the duration of antibiotic treatment was significantly shorter for 24 UTI-patients receiving procalcitonin-guided treatment compared to 18 UTI control-group patients [4]. However further investigations are scarce. Other biomarkers may also be of interest, such as mid-regional proadrenomedullin (MR-proADM), which has been shown to be increased in the early stages of progression towards multiple organ failure [5], and is of value in predicting a complicated course of treatment and the requirement for ICU admission [6-8].

This secondary analysis of the earlier FUTIRST trial [1] therefore hypothesized that procalcitonin measurement on days 0 and 3 could more accurately identify patients at risk of treatment failure and in need a prolonged course of antibiotics compared to either MR-proADM or C-reactive protein.

\section{Methods}

\section{Design and study population}

This was a secondary analysis of a randomized, placebocontrolled trial involving patients presenting with febrile urinary tract infection (fUTI) at the emergency departments (ED) of 7 hospitals and 32 primary health care centres in the Netherlands, between November 2008 and May 2013, as described previously [1, 9]. This trial enrolled consecutive adult patients with a presumptive diagnosis of community-acquired fUTI established by a primary care or emergency physician, who met the following criteria: fever of $\geq 38.2^{\circ} \mathrm{C}$ and/or a history of feeling feverish with shivering or rigors in the past $24 \mathrm{~h}$, one or more symptoms suggestive of UTI (i.e. dysuria, frequency, urgency, perineal or suprapubic pain, costovertebral tenderness, or flank pain), and positive urine nitrate test and/or pyuria (positive leucocyte esterase test or $>5$ leucocytes per high-power field in a centrifuged sediment). Exclusion criteria for study entry were as follows: known allergy to fluoroquinolones, pregnancy or lactation, polycystic kidney disease, permanent renal replacement therapy and kidney transplantation. Patients enrolled with fUTI but not randomized to trial medication remained in the observational part of the study to assess outcome [1]. In the current study, all patients participating in both observational and interventional part of the trial were included, except those without a baseline blood sample available for biomarker analysis, without a 10-18 days post-treatment visit, and patients who needed prolonged treatment because of chronic bacterial prostatitis.

The study protocol was approved by the local Ethical Committee, and written informed consent was obtained from all participants. The trial was registered at ClinicalTrials.gov (NCT00809913; December 16, 2008) and trialregister.nl (NTR1583; December 19, 2008).

\section{Procedures}

Within 24-48 h of notification, qualified research nurses collected clinical data and laboratory values by standardized questionnaires. The decision whether to treat as outpatient or inpatient was left to the discretion of the attending physician. Outpatients started with the first week of open label ciprofloxacin $500 \mathrm{mg}$ twice daily. In hospitalized patients, the treating physician could administer empirical intravenous antibiotics at the start of treatment according to local policy (in all participating centres: a ß-lactam antibiotic \pm aminoglycoside). These patients were switched to open label ciprofloxacin as soon as was deemed possible. Randomization between the second week ciprofloxacin and placebo twice daily 
was initiated once the results of the urine culture became available on the third or fourth day after inclusion. In patients who could not be randomized (e.g. due to ciprofloxacin resistance), the choice of antibiotic agent and treatment duration was left at the discretion of the treating physician [1].

All patients were contacted in person on day 3 (3-4 days after start of treatment) and day 30 (10-18 days post-treatment) after enrolment, and by phone on day 90 (70-84 days post-treatment) to assess clinical outcome. EDTA plasma samples were collected, centrifuged and stored at $-80^{\circ} \mathrm{C}$ within $2 \mathrm{~h}$ of patient enrolment. MR-proADM and PCT were batch-measured in a blinded fashion by TRACE technology (Time Resolved Amplified Cryptate Emission) using a new sandwich immunoassay (Kryptor Compact Plus Analyzer, BRAHMS, Hennigsdorf, Germany), with a limit of detection of 0.05 $\mathrm{nmol} / \mathrm{L}$ and $0.02 \mathrm{ng} / \mathrm{L}$, respectively. Further details on randomization, trial medication and study procedures have been published previously $[1,9]$.

\section{Outcome measure}

The biomarkers PCT and MR-proADM were evaluated for their ability to predict the clinical cure. Clinical cure was assessed on the day 30 visit (10-18 days post-treatment), and was defined as survival with absence of fever and a resolution of UTI symptoms (either absence of symptoms or at least 2 points improvement on a $0-5$ point severity score scale), without additional antimicrobial therapy (for relapse of UTI) [1]. Patients who did not meet the criteria of clinical cure at day 30 , were considered to have clinical treatment failure. Long term clinical cure was assessed at the 90-day post-treatment interview.

\section{Statistical analysis}

Descriptive statistics are expressed as frequencies (percentage), means with standard deviation (SD) or as medians with interquartile range (IQR), as appropriate. Univariate analysis was performed using ANOVA, student's t-test or Mann-Whitney U test where appropriate for continuous variables and Chi-square test for categorical variables. Non-parametric tests were used to analyse biomarkers.

Analyses were performed in the total patient population and in 2 subgroups based on treatment duration. Kendall's rank tau-b was used to investigate correlations between biomarker levels and clinical parameters. Finally, area under the receiver operating characteristic (AUROC) curves with 95\% confidence intervals (CI) were calculated to assess the prognostic ability of PCT and MR-proADM.

A $p$-value of $<0.05$ was considered to indicate statistical significance. SPSS software (SPSS Inc. Chicago, version 23.0) was used for statistical analysis.

\section{Results}

A total of 249 patients with a presumptive diagnosis of fUTI were analysed (details provided in Fig. 1). Patient characteristics in terms of urologic history, comorbidities and presenting symptoms are outlined in Table 1. Of these, $165(66 \%)$ were randomly assigned to receive antimicrobial treatment for either $7(N=85)$ or $14(N=80)$ days; in the remaining patients the treatment duration was left at the discretion of the treating physician. The majority of patients $(N=175 ; 70 \%)$ were included at the $E D$, and 73 (29\%) patients undergoing existing antimicrobial treatment prior to presentation. Patients had an average age of 60 (45-73) years, with females comprising the majority of enrolled patients $(N=148 ; 59 \%)$.

The clinical cure rate was high $(N=229 ; 92 \%)$ and did not differ significantly between randomized and non-randomized patients ( $94 \%$ vs. $88 \%$, respectively). A total of 20 patients did not reach the endpoint of clinical cure, due to persistence or recurrence of UTI symptoms $(\mathrm{N}=8)$, or to the use of additional antibiotics for relapse of UTI $(N=12)$, assessed on the day 30 visit. No significant differences were seen in the clinical characteristics between patients with treatment success or failure. In addition, mean treatment duration was similar (11 days) in both patients with clinical cure and clinical failure (Table 2).

Median biomarker concentrations across the total patient population were as follows: PCT: 0.40 [0.12-1.54] $\mathrm{ug} / \mathrm{mL}$; MR-proADM: 0.89 [0.63-1.28] $\mathrm{nmol} / \mathrm{L} ;$ and CRP: 118 (52-205) mg/L.

\section{Prediction of treatment outcome Total patient population}

Concentrations of PCT, MR-proADM and CRP, measured at presentation, did not differ between patients with clinical cure or treatment failure (Fig. 2 and Table 3). As shown in Table 3, biomarker levels also did not predict treatment failure when measured after 3 days of treatment. We also assessed cut-offs for PCT as previously used in other studies, such as $0.25 \mu \mathrm{g} / \mathrm{mL}[10,11]$ and a decrease of PCT concentration by $80 \%$ [4].

ROC analyses were further performed to define the prognostic accuracy of the different biomarkers for predicting treatment outcome. Based on the calculated AUCs, none of the biomarkers had any predictive value for treatment success (Fig. 3). Findings were similar in a selection of patients with culture proven UTI $(N=183)$; and regarding the long term clinical cure in all patients (data not shown).

\section{Patients treated for 7 days}

We assessed the group of patients who received 7 days of antimicrobial treatment separately, because treatment success was lower as compared to those treated for 14 


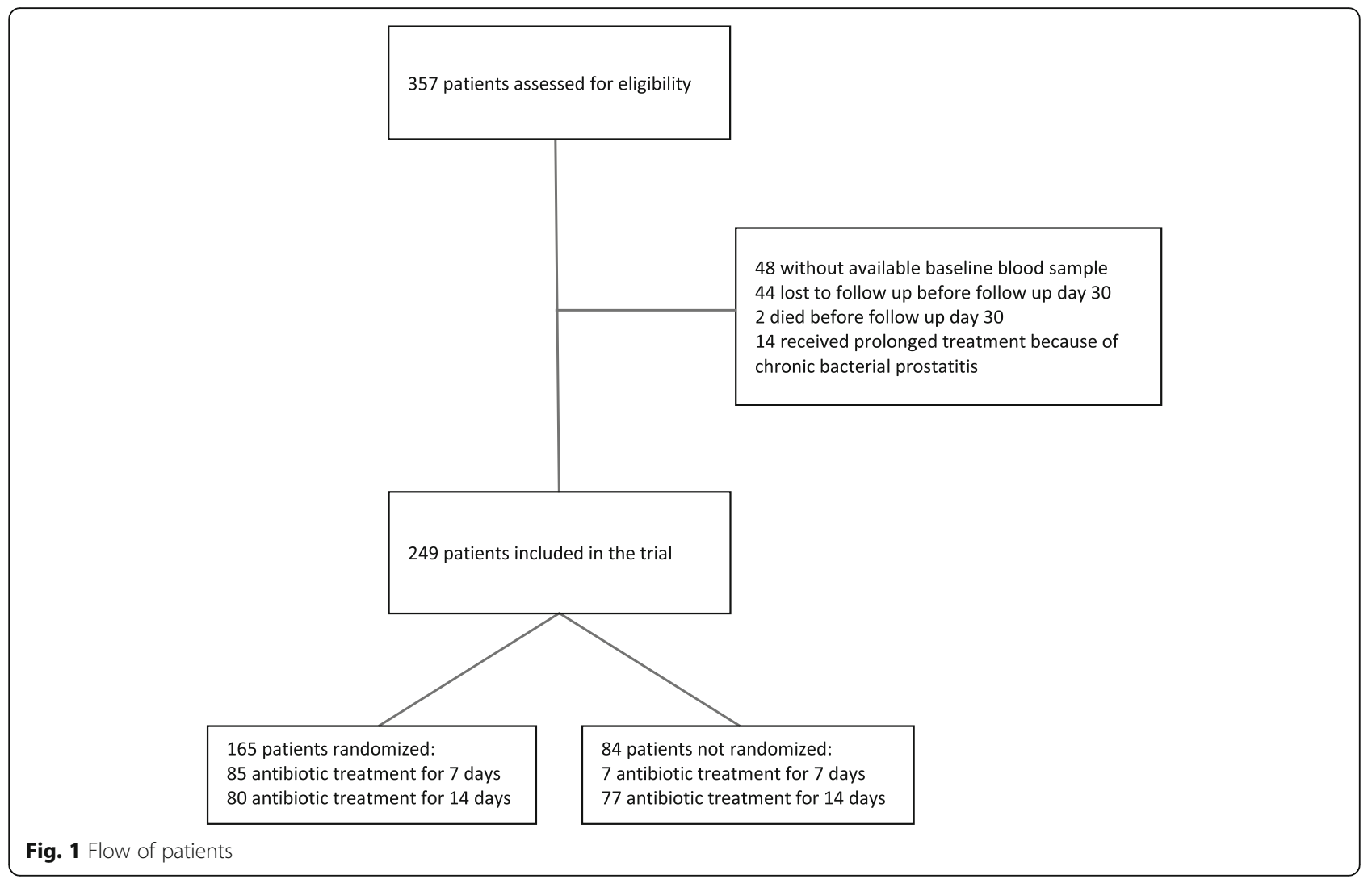

days ( $89 \%$ versus $94 \%$, respectively, $p=0.21$ ). Again, no difference was seen in levels of PCT, MR-proADM and CRP between patients with clinical cure or treatment failure (Table 3).

\section{Correlation between biomarkers and clinical parameters Procalcitonin}

PCT concentrations at presentation were positively correlated with bacteraemia $(\tau=0.33, p<0.001)$ and presence of shaking chills $(\tau=0.25, p<0.001)$. Although significant, correlations with initial intravenous treatment, length of hospital admission, time to defervescence, ICU admission, temperature, heart rate and confusion at presentation were weak $(\tau<0.20)$. Furthermore, PCT increased slightly with age $(\tau=0.12, p<$ 0.01 ) and was not correlated to comorbidity. Median PCT concentrations measured after 3 days of treatment showed a similar trend, whereas the decrease of PCT concentration was not correlated to clinical parameters.

\section{Mid-regional proadrenomedullin}

MR-proADM levels at presentation were positively correlated with length of hospital stay $(\tau=0.40, p<0.001)$, bacteraemia $(\tau=0.33, \mathrm{p}<0.001)$, initial intravenous treatment $(\tau=0.22, p<0.001)$ and time to defervescence ( $\tau=0.21, p<0.001)$. Weak, though significant correlation was seen with temperature, need for ICU admission, confusional state at presentation and the presence of shaking chills $(\tau<0.20)$. Compared to PCT, MRproADM showed a stronger correlation with age $(\tau=$ $0.49, p<0.001$ ), and was weakly correlated to pre-existing comorbidity (diabetes mellitus, urinary tract disorder and chronic renal insufficiency). Again, after 3 days of treatment, correlations were similar. Decreasing MR-proADM concentrations between presentation and day 3 were correlated to bacteraemia $(\tau=0.22, p<0.001)$ and the presence of shaking chills at presentation $(\tau=$ $0.22, p<0.001)$.

\section{C-reactive protein}

CRP was only weakly positively correlated to time to defervescence ( $\tau=0.13, p=0.02$ ) and weakly negatively correlated to temperature $(\tau=-0.11, p=0.04)$ and mean arterial pressure $(\tau=-0.13 p=0.02)$. No correlations were found between CRP and other parameters, such as age or comorbidity.

\section{Discussion}

In the current study we assessed the ability of the biomarkers PCT, MR-proADM and CRP in predicting treatment failure in a randomized trial of antibiotic treatment duration (i.e. 7 versus 14 days) in patients with febrile urinary tract infection. We hypothesized that the biomarker signature, as an objective laboratory proxy of 
Table 1 Baseline characteristics of 249 patients with febrile urinary tract infection

\begin{tabular}{|c|c|}
\hline & All patients $(n=249)$ \\
\hline Age (years) & $60(45-73)$ \\
\hline Male sex & $101(41)$ \\
\hline \multicolumn{2}{|l|}{ Urologic history } \\
\hline Indwelling urinary catheter & $7(3)$ \\
\hline Urinary tract disorder ${ }^{a}$ & $75(30)$ \\
\hline Recurrent $U \mathrm{TI}^{\mathrm{b}}$ & $57(23)$ \\
\hline \multicolumn{2}{|l|}{ Comorbidity } \\
\hline Diabetes mellitus & $33(13)$ \\
\hline Malignancy & $17(7)$ \\
\hline Heart failure & $21(8)$ \\
\hline Cerebrovascular disease & $13(5)$ \\
\hline Chronic renal insufficiency & $9(4)$ \\
\hline COPD & $27(11)$ \\
\hline Immunocompromised & $15(6)$ \\
\hline \multicolumn{2}{|l|}{ Presentation } \\
\hline At emergency department & $175(70)$ \\
\hline Antibiotic pretreatment & $73(29)$ \\
\hline Fever duration, hours & $36(19-72)$ \\
\hline Dysuria & $188 / 243(77)$ \\
\hline Flank pain & $159 / 245(65)$ \\
\hline Suprapubic pain & $127 / 242(52)$ \\
\hline Perineal pain & $11 / 241(5)$ \\
\hline Systolic BP, mmHg & $130(116-146)$ \\
\hline Diastolic BP, mmHg & $74(64-84)$ \\
\hline Heart rate, beats/minute & $94(80-107)$ \\
\hline \multicolumn{2}{|l|}{ Cultures } \\
\hline Positive urine culture & $171(69)$ \\
\hline Escherichia coli & 143/171 (84) \\
\hline Positive blood culture & $45 / 240(19)$ \\
\hline Positive urine and/or blood culture & $183(73)$ \\
\hline
\end{tabular}

Data presented as number (\%) or median (IQR).

$B P$ blood pressure, $A B$ antibiotics, TMP/SMX trimethoprim-sulfamethoxazole.

a any functional or anatomical abnormality of urinary tract except

urinary catheter

$b_{\geq 3}$ UTIs in past 12 months or $\geq 2$ UTIs in past 6 months

physical status in fUTI, would help the physician in guiding the length of antibiotic therapy. In our study, however, neither PCT nor MR-proADM or CRP were distinctive in identifying upfront those patients at risk for a treatment failure, i.e. who might benefit from a more prolonged antibiotic treatment.

Overall treatment success was high: $94 \%$ of the randomized 165 and $88 \%$ of 84 non-randomized patients who all were given an identical follow-up reached the endpoint of clinical cure. Of the biomarkers, PCT and MR-proADM were significantly correlated to clinical parameters such as fever, blood pressure and subjective complaints that represent the acute febrile illness of invasive urinary tract infection. Also, the biomarker signature of PCT and MR-proADM correlated with severity of disease, such as presence of bacteraemia and need for initial administration of antibiotics intravenously rather than orally. Finally, the course of biomarkers over the first three days correlated with signs of clinical recovery, such as time to defervescence, intensity of subjective complaints and length of hospital stay. As opposed to PCT and MR-proADM, the currently popular biomarker CRP did not display any correlation to relevant clinical parameters like course of fever or bacteraemia.

The biomarkers we chose are in current use as predictors of morbidity and mortality in a variety of conditions. Procalcitonin is a precursor hormone of calcitonin and is upregulated by cytokines released in response to bacterial infection [4]. MR-proADM has been detected in a variety of tissues, including heart, vessels and the kidneys and has both immune modulating and vasodilating properties [2]. Levels of MR-proADM are elevated in sepsis, contributing to hypotension in these patients. Thus, procalcitonin has shown to be a marker of bacteraemia in patients with febrile UTI [12-14], whereas mid-regional proadrenomedullin (MR-proADM) is a predictor of a complicated course of disease, the need for ICU admission, and mortality [6, 7].

PCT-based algorithms have been developed to aid decisions on the initiation and/or discontinuation of antibiotics in patients with acute respiratory tract infections and in critically ill patients admitted to the intensive care ward [15]. In these specific patient groups, the use of PCT has been shown to be a powerful tool in antibiotic stewardship [16]. Although in the intensive care studies patients with infections originating from the urinary tract were included, numbers were small (e.g. $7 \%$ of patients in the PRORATA trial, $3 \%$ of patients in the SAPS trial) and these results cannot directly be extrapolated to patients with community-acquired urinary tract infections $[4,17]$.

Elevation of these biomarkers is a reflection of the systemic inflammatory response to bacterial invasion. This inflammatory response coincides with acute illness, and often in a measurable deviation of vital signs, such as a raised temperature and decreased blood pressure. In daily clinical practice, severity of the acute illness is assessed on basis of history, comorbidity and on severity of local and vital signs. It has become clear that severity of illness can also be more objectively expressed in biomarkers, which individually are associated with complicated course of disease, such as bacteraemia, need for ICU admission, time to defervescence and length of hospital stay. Although PCT and proADM were correlated to these clinical outcome parameters in our patients, 
Table 2 Characteristics of patients with clinical cure and clinical failure

\begin{tabular}{|c|c|c|c|}
\hline & Clinical cure $(n=229)$ & Clinical failure $(n=20)$ & $P$ value \\
\hline Age (years) & $60(45-73)$ & $56(46-71)$ & 0.412 \\
\hline Male sex & $94(41)$ & $7(35)$ & 0.597 \\
\hline \multicolumn{4}{|l|}{ Urologic history } \\
\hline Indwelling urinary catheter & $7(3)$ & 0 & 0.428 \\
\hline Urinary tract disorder ${ }^{a}$ & $71(31)$ & $4(20)$ & 0.304 \\
\hline Recurrent UTI ${ }^{\mathrm{b}}$ & $50(22)$ & $7(35)$ & 0.191 \\
\hline \multicolumn{4}{|l|}{ Comorbidity } \\
\hline Diabetes mellitus & $32(14)$ & $1(5)$ & 0.256 \\
\hline Malignancy & $16(7)$ & $1(5)$ & 0.735 \\
\hline Heart failure & $21(9)$ & 0 & 0.157 \\
\hline Cerebrovascular disease & $12(5)$ & $1(5)$ & 0.963 \\
\hline Chronic renal insufficiency & $8(3)$ & $1(5)$ & 0.729 \\
\hline COPD & $27(11)$ & $2(10)$ & 0.899 \\
\hline Immunocompromised & $14(6)$ & $1(5)$ & 0.841 \\
\hline \multicolumn{4}{|l|}{ Presentation } \\
\hline At emergency department & $160(70)$ & $15(75)$ & 0.630 \\
\hline Antibiotic pre-treatment & $71(31)$ & $2(10)$ & 0.048 \\
\hline Fever duration, hours & $36(18-72)$ & $48(24-120)$ & 0.279 \\
\hline Dysuria & 175/224 (78) & 13/19 (68) & 0.332 \\
\hline Flank pain & $142 / 225(63)$ & $17(85)$ & 0.049 \\
\hline Suprapubic pain & 116/222 (52) & $11(55)$ & 0.814 \\
\hline Perineal pain & $11 / 222(5)$ & 0/19 (0) & 0.321 \\
\hline Systolic BP, mmHg & $130(116-146)$ & $130(115-150)$ & 0.753 \\
\hline Diastolic BP, mmHg & $74(63-85)$ & $72(68-83)$ & 0.585 \\
\hline Heart rate, beats/minute & $93(80-107)$ & $96(78-110)$ & 0.695 \\
\hline \multicolumn{4}{|l|}{ Cultures } \\
\hline Positive urine culture & $158(69)$ & $13(65)$ & 0.712 \\
\hline Escherichia coli & 133/158 (84) & 10/13 (77) & 0.497 \\
\hline Positive blood culture & 42/220 (19) & $3(15)$ & 0.654 \\
\hline Positive urine and/or blood culture & $169(74)$ & $14(70)$ & 0.712 \\
\hline \multicolumn{4}{|l|}{ Treatment } \\
\hline Short antimicrobial treatment (7 days) & $82(36)$ & $10(50)$ & 0.207 \\
\hline Days of $A B$, mean (SD) & $11(3.3)$ & $11(3.5)$ & 0.252 \\
\hline Ciprofloxacin & $208(91)$ & $18(90)$ & 0.902 \\
\hline Amoxicillin ( \pm clavulanic acid) & $11(5)$ & $2(10)$ & 0.316 \\
\hline TMP/SMX & $5(2)$ & 0 & 0.504 \\
\hline Other ${ }^{c}$ & $5(2)$ & 0 & 0.504 \\
\hline Initial intravenous dose(s) of $A B$ & $135(59)$ & $12(60)$ & 0.927 \\
\hline Randomized & $155(68)$ & $10(50)$ & 0.109 \\
\hline Outpatient treatment & $89(39)$ & $7(35)$ & 0.733 \\
\hline
\end{tabular}

Data presented as number (\%) or median (IQR)

$B P$ blood pressure, $A B$ antibiotics, TMP/SMX trimethoprim-sulfamethoxazole

a any functional or anatomical abnormality of urinary tract except urinary catheter

${ }^{b} \geq 3$ UTIs in past 12 months or $\geq 2$ UTIs in past 6 months

cefuroxime iv $n=2$, meropenem iv $n=1$, moxifloxacin $n=1$, flucloxacillin $n=1$ 

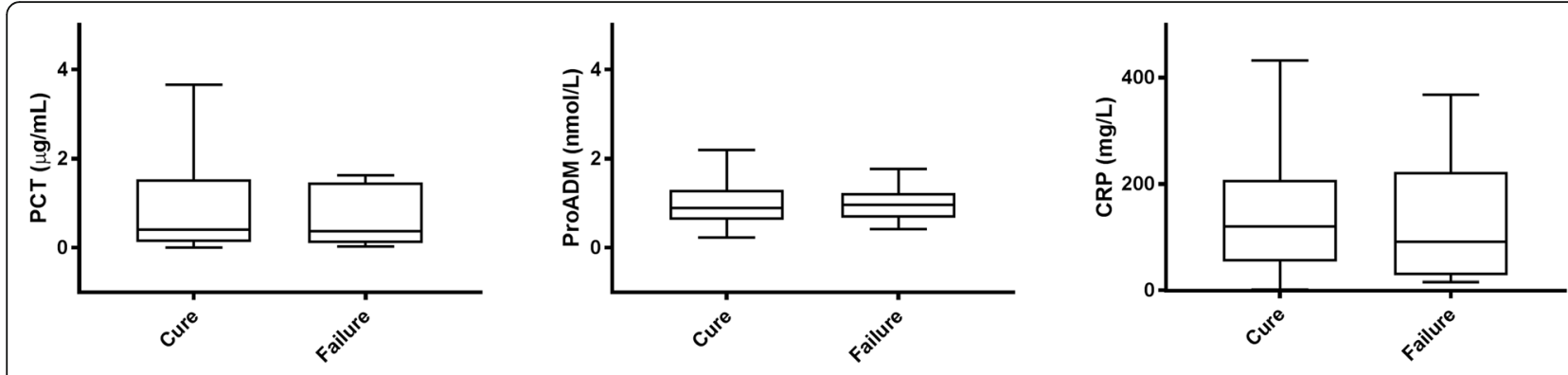

Fig. 2 Levels of PCT, MR-proADM and CRP measured at presentation in patients with clinical cure and failure

Table 3 Biomarkers in patients with clinical cure and clinical failure

\begin{tabular}{|c|c|c|c|}
\hline & Clinical cure & Clinical failure & $P$ value \\
\hline All patients $(n=249)$ & $n=229$ & $n=20$ & \\
\hline \multicolumn{4}{|l|}{ At presentation } \\
\hline PCT & $0.40(0.13-1.54)$ & $0.36(0.10-1.46)$ & 0.749 \\
\hline$P C T>0.25$ & $136(59 \%)$ & $12(60 \%)$ & 0.957 \\
\hline CRP & $120(53-211)$ & $90(27-223)$ & 0.851 \\
\hline proADM & $0.89(0.62-1.30)$ & $0.86(0.67-1.20)$ & 0.948 \\
\hline \multicolumn{4}{|l|}{ Day 3} \\
\hline PCT & $0.18(0.07-0.87)$ & $0.12(0.04-0.69)$ & 0.667 \\
\hline $\mathrm{PCT} \leq 0.25$ & $122(60 \%)$ & $12(67 \%)$ & 0.568 \\
\hline$P C T \leq 0.25$ or $P C T$ decline $\geq 80 \%$ & $144(71 \%)$ & $14(78 \%)$ & 0.519 \\
\hline proADM & $0.66(0.50-0.91)$ & $0.63(0.51-0.78)$ & 0.667 \\
\hline Short treatment $(n=92)$ & $n=82$ & $n=10$ & \\
\hline \multicolumn{4}{|l|}{ At presentation } \\
\hline PCT & $0.34(0.10-1.68)$ & $0.36(0.11-1.79)$ & 0.980 \\
\hline $\mathrm{PCT}>0.25$ & $47(57 \%)$ & $6(60 \%)$ & 0.871 \\
\hline CRP & $123(53-194)$ & $77(15-142)$ & 0.231 \\
\hline proADM & $0.76(0.56-1.04)$ & $0.85(65-1.25)$ & 0.360 \\
\hline \multicolumn{4}{|l|}{ Day 3} \\
\hline PCT & $0.12(0.06-0.42)$ & $0.13(0.09-0.63)$ & 0.794 \\
\hline $\mathrm{PCT} \leq 0.25$ & $51(66 \%)$ & $7(78 \%)$ & 0.484 \\
\hline$P C T \leq 0.25$ or $P C T$ decline $\geq 80 \%$ & $62(80 \%)$ & $8(89 \%)$ & 0.542 \\
\hline proADM & $0.56(0.47-0.76)$ & $0.59(0.53-0.74)$ & 0.679 \\
\hline Long treatment $(n=157)$ & $n=147$ & $n=10$ & \\
\hline \multicolumn{4}{|l|}{ At presentation } \\
\hline PCT & $0.48(0.15-1.49)$ & $0.48(0.09-3.13)$ & 0.826 \\
\hline $\mathrm{PCT}>0.25$ & $89(60 \%)$ & $6(60 \%)$ & 0.973 \\
\hline CRP & $119(52-224)$ & $181(37-263)$ & 0.531 \\
\hline proADM & $0.98(0.67-1.44)$ & $0.86(0.62-1.21)$ & 0.495 \\
\hline \multicolumn{4}{|l|}{ Day 3} \\
\hline PCT & $0.22(0.08-1.08)$ & $0.08(0.04-0.72)$ & 0.358 \\
\hline$P C T \leq 0.25$ & $71(56 \%)$ & $5(56 \%)$ & 0.984 \\
\hline$P C T \leq 0.25$ or $P C T$ decline $\geq 80 \%$ & $82(65 \%)$ & $6(67 \%)$ & 0.899 \\
\hline proADM & $0.74(0.53-1.03)$ & $0.74(0.47-1.02)$ & 0.609 \\
\hline
\end{tabular}

Data presented as median (IQR) or number (\%)

CRP at presentation missing: $n=41$ in short treatment and $n=41$ in long treatment 

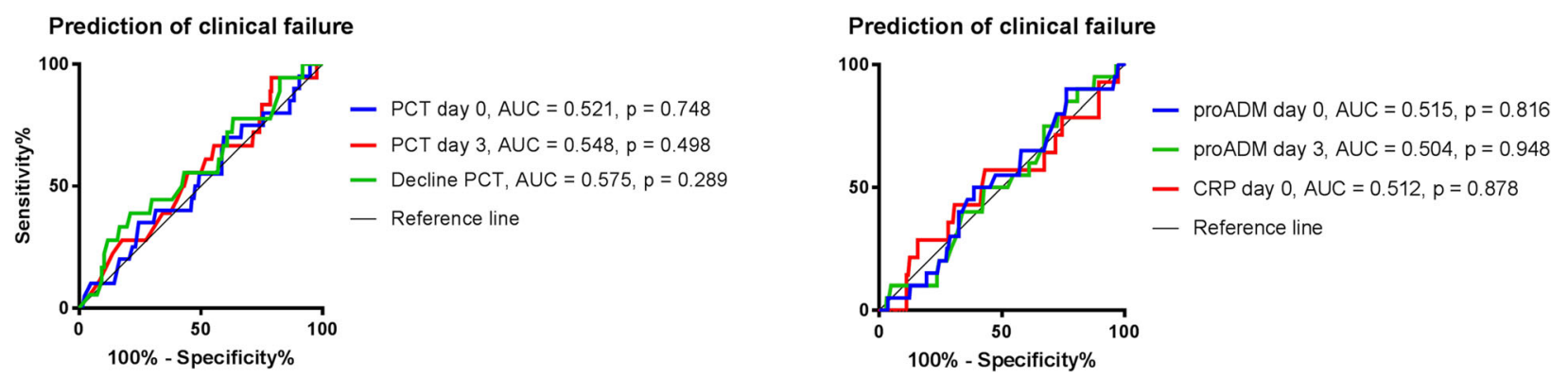

Fig. 3 Biomarker accuracy in the prediction of treatment outcome

they did not help predict outcome of the otherwise standardized antibiotic treatment, irrespective of a treatment duration of 7 or 14 days. The most likely reason is that all patients were treated for at least 7 days after which treatment success was high already (i.e., 89\%). Thus, the lack of predictive value for treatment guidance is likely explained by the fact that historically, empiric treatment duration is based on the anticipated time to clinical recovery, while taking into account the inter-individual variety in severity of acute illness at the start of treatment (i.e. practically, adding some days of treatment to average recovery time). Thus, in our patients, the median time to defervescence was 2.0 (IQR 1-3) days while randomization for short or prolonged treatment duration did not start until day 7 . In other words, one could have predicted that our biomarker approach might have been successful in guiding treatments up to one or a few days after clinical recovery, but it lacked the ability to do so after the minimum 7 days of treatment, by which a strong margin surpasses the time for the biomarker signature to return to normal. Differences between patients in severity of illness at presentation and corresponding biomarker levels are likely to have normalized after 7 days of treatment, and definitely after 14 days. It should be noted, that antimicrobial resistance played no role in our study; patients with a ciprofloxacin resistant uropathogen were excluded from randomization and received effective therapy based on culture results.

In the original study we described that although 7 days of treatment was inferior to 14 days in male patients in terms of short term clinical cure, there was no difference in the requirement for antibiotic retreatment for UTI during longer follow up (90 days) in both women and men [1].

A retrospective analysis of a large database of male veterans also found that treatment duration longer than 7 days was not associated with a reduction of UTI recurrence [18]. In this study, UTI recurrence was independently associated with age and comorbidity. It is likely that a subgroup of patients can be treated with an even shorter course of antibiotics than 7 days. Hypothetically, within the current time frame of treatment, there is a moment when the patient has recovered from the acute illness and the biomarker levels have dropped below a certain cut-off, likely leaving room for further limitation of treatment duration. It stands to reason that the time to this recovery is defined by host related factors, and therefore differs between patient subgroups. Biomarkers have the potential to objectively identify the optimal moment for cessation of therapy, irrespective of patient characteristics. Unfortunately, as reasoned above, our study design did not allow for further discrimination.

The main strength of this trial is its pragmatic nature reflecting daily clinical practice. We randomized patients between the up until then standard treatment duration for fUTI of 14 days and half of that length (7 days), and included enough subjects to demonstrate non-inferiority. We enrolled consecutive patients with fUTI, irrespective of age, gender and underlying medical condition. Different biomarker concentrations were available at the day of presentation and after three days of treatment, each representing different aspects of the physiological condition of the acute illness.

Limitations of our study include the design in randomizing patients between 7 and 14 days, not allowing for analysis of biomarker value in shorter antibiotic treatment. This was unforeseen, because at the start of the trial 14 days of therapy were standard clinical practice, and reduction to 7 days was already a great improvement. Furthermore, our sample size may have been too small to exclude a type II error.

To our knowledge, only one study addressing biomarkerguided antibiotic therapy in community acquired urinary tract infection has been published [10]. In this study, Drozdov et al. randomized both patients with cystitis $(N=36)$ and febrile urinary tract infections $(N=84)$ between PCT-guided or standard treatment. Overall, antibiotic exposure was reduced whereas adverse outcomes (clinical recurrence and rehospitalization rate) were similar in both groups. In the subgroup analysis of patients with fUTI/pyelonephritis, PCT-guided duration of antibiotic therapy was significantly shorter than standard care (7.5 vs. 11.0 days). 
In bacteraemic patients, recurrence rate (56\% vs. $16 \%)$ and persistent infection after treatment $(13 \%$ vs. $6 \%)$ were higher in the PCT guided group, although the distribution of patients with bacteraemia was not well balanced between the groups ( $45 \%$ vs. $21 \%)$ and numbers were too small to reach significance. Although this study by Drozdov et al. is limited by the small sample size and heterogenicity of patients, it supports the potential use of PCT-based approach in patients with community acquired fUTI to safely reduce antibiotic consumption.

Future interventional studies should be conducted to examine if PCT-guided duration of antibiotic treatment can indeed aid in distinguishing a subgroup of patients with fUTI who can be treated with antibiotics for even shorter than 7 days, i.e. those with rapid normalization of elevated biomarkers, in order to further decrease antibiotic exposure and limit development of antimicrobial resistance.

In conclusion, although the biomarkers PCT and MR-proADM are correlated to clinical parameters indicating disease severity, they did not predict treatment outcome in patients with community acquired febrile urinary tract infection, who are treated for 7 or 14 days with ciprofloxacin. In our secondary analysis, CRP had no added value in the management of fUTI.

\section{Abbreviations \\ AB: Antibiotics; AUC: Area under the curve; BP: Blood pressure; Cl: Confidence interval; CRP: C-reactive protein; ED: Emergency department: fUTI: Febrile urinary tract infection; ICU: Intensive care unit; IQR: Interquartile range; MR-proADM: mid-regional proadrenomedullin; PCT: Procalcitonin; ROC: Receiver operating characteristics curves; SD: Standard deviation; TMP/ SMX: Trimethoprim-sulfamethoxazole; TRACE: Time Resolved Amplified Cryptate Emission}

\section{Acknowledgements}

The authors thank the patients, research nurses, emergency room physicians, nurses and laboratory staff for their cooperation. We thank Thermo Fisher Scientific / Brahms, Hennigsdorf, Germany for measurement of ProADM and $\mathrm{PCT}$ in the blinded samples.

\section{Funding}

This study was supported by unrestricted grands by ZonMW (projectnumber 171101003), the Bronovo Research Foundation and the Franje1 Foundation. There was no role of the funding organizations in design and conduct of the study; collection, management, analysis, and interpretation of the data; and preparation or approval of the manuscript; or decision to submit the manuscript for publication.

\section{Availability of data and materials \\ The authors affirm that the manuscript is an honest, accurate, and transparent account of the study being reported; that no important aspects of the study have been omitted; and that any discrepancies are disclosed. The datasets generated and analysed during the current study are not publicly available as patient's consent was not obtained for data sharing. However, because the presented data are anonymized, and the risk of identification is low, data are available on reasonable request from the corresponding author.}

\section{Authors' contributions}

JES and JVD conceived the study, designed the trial, and obtained research funding. JES, CVN, WvdS and JvD supervised the conduct of the trial and data collection. JES, WvdS, CVN, NMD, EMSL, TK, HCA and JWvtW undertook recruitment of participating centers and patients. TJKvdR handled all the samples in the laboratory, and DCW was responsible for measurement of ProADM and PCT in the blinded samples. JES and JVD analysed the data. JES drafted the manuscript, and all authors contributed substantially to its revision. JVD takes responsibility for the paper as a whole. All authors read and approved the final manuscript.

\section{Ethics approval and consent to participate}

The study protocol was primarily approved by the Medical Ethics Committee of the Leiden University Medical Center (protocol P08.65) and subsequently by all scientific boards of all participating centers. All patients have given written informed consent.

\section{Consent for publication}

Not Applicable.

\section{Competing interests}

Darius Wilson is employed by Thermo Fisher Scientific / Brahms, Hennigsdorf, Germany. All other authors declare that they have no competing interests.

\section{Publisher's Note}

Springer Nature remains neutral with regard to jurisdictional claims in published maps and institutional affiliations.

\section{Author details}

'Department of Infectious Diseases, Leiden University Medical Center, POBox 9600, 2300 RC Leiden, the Netherlands. ${ }^{2}$ Department of Internal Medicine, Haga Hospital, The Hague, The Netherlands. ${ }^{3}$ Thermo Fisher Scientific, Hennigsdorf, Germany. ${ }^{4}$ Department of Internal Medicine, Alrijne Hospital, Leiderdorp, The Netherlands. ${ }^{5}$ Department of Internal Medicine, $\mathrm{MCH}$, The Hague, The Netherlands. ${ }^{6}$ Department of Internal Medicine, Groene Hart Hospital, Gouda, The Netherlands. ${ }^{7}$ Department of Internal Medicine, Alrijne Hospital, Leiden, The Netherlands.

Received: 25 September 2018 Accepted: 7 February 2019

Published online: 14 February 2019

\section{References}

1. van Nieuwkoop C, van der Starre WE, Stalenhoef JE, van Aartrijk AM, van der Reijden TJ, Vollaard AM, Delfos NM, van 't Wout JW, Blom JW, Spelt IC et al: Treatment duration of febrile urinary tract infection: a pragmatic randomized, double-blind, placebo-controlled non-inferiority trial in men and women. BMC Med 2017, 15(1):70

2. Christ-Crain M, Morgenthaler NG, Stolz D, Muller C, Bingisser R, Harbarth S, Tamm M, Struck J, Bergmann A, Muller B. Pro-adrenomedullin to predict severity and outcome in community-acquired pneumonia [ISRCTN04176397]. Crit Care. 2006;10(3):R96.

3. Schuetz P, Muller B, Christ-Crain M, Stolz D, Tamm M, Bouadma L, Luyt CE, Wolff $M$, Chastre J, Tubach F, et al. Procalcitonin to initiate or discontinue antibiotics in acute respiratory tract infections. Cochrane Database Syst Rev. 2012:9 CD007498

4. Bouadma L, Luyt CE, Tubach F, Cracco C, Alvarez A, Schwebel C, Schortgen F, Lasocki S, Veber B, Dehoux M, et al. Use of procalcitonin to reduce patients' exposure to antibiotics in intensive care units (PRORATA trial): a multicentre randomised controlled trial. Lancet. 2010;375(9713):463-74.

5. Elke G, Bloos F, Wilson DC, Brunkhorst FM, Briegel J, Reinhart K, Loeffler M, Kluge S, Nierhaus A, Jaschinski U, et al. The use of mid-regional proadrenomedullin to identify disease severity and treatment response to sepsis - a secondary analysis of a large randomised controlled trial. Crit Care. 2018;22(1):79.

6. van der Starre WE, Zunder SM, Vollaard AM, van NC, Stalenhoef JE, Delfos NM, van't Wout JW, Spelt IC, Blom JW, Leyten EM, et al. Prognostic value of pro-adrenomedullin, procalcitonin and C-reactive protein in predicting outcome of febrile urinary tract infection. Clin Microbiol Infect. 2014;20(10): $1048-54$.

7. Andaluz-Ojeda D, Nguyen HB, Meunier-Beillard N, Cicuendez R, Quenot JP, Calvo D, Dargent A, Zarca E, Andres C, Nogales L, et al. Superior accuracy of mid-regional proadrenomedullin for mortality prediction in sepsis with varying levels of illness severity. Ann Intensive Care. 2017;7(1):15. 
8. Stalenhoef JE, van Nieuwkoop C, Wilson DC, van der Starre WE, Delfos NM, Leyten EMS, Koster T, Ablij HC, Willem Van't Wout JJ, van Dissel JT: Biomarker guided triage can reduce hospitalization rate in community acquired febrile urinary tract infection. J Infect. 2018;77(1):18-24. https://doi. org/10.1016/j.jinf.2018.05.007. Epub 2018 May 26

9. van Nieuwkoop C, van't Wout JW, Assendelft WJ, Elzevier HW, Leyten EM, Koster T, Wattel-Louis GH, Delfos NM, Ablij HC, Kuijper EJ, et al. Treatment duration of febrile urinary tract infection (FUTIRST trial): a randomized placebocontrolled multicenter trial comparing short (7 days) antibiotic treatment with conventional treatment (14 days). BMC Infect Dis. 2009;9:131.

10. Drozdov D, Schwarz S, Kutz A, Grolimund E, Rast AC, Steiner D, Regez K Schild U, Guglielmetti M, Conca A, et al. Procalcitonin and pyuria-based algorithm reduces antibiotic use in urinary tract infections: a randomized controlled trial. BMC Med. 2015;13:104.

11. Christ-Crain M, Jaccard-Stolz D, Bingisser R, Gencay MM, Huber PR, Tamm M, Muller B. Effect of procalcitonin-guided treatment on antibiotic use and outcome in lower respiratory tract infections: cluster-randomised, singleblinded intervention trial. Lancet. 2004;363(9409):600-7.

12. van Nieuwkoop C, Bonten TN, van't Wout JW, Kuijper EJ, Groeneveld GH, Becker MJ, Koster T, Wattel-Louis GH, Delfos NM, Ablij HC, et al. Procalcitonin reflects bacteremia and bacterial load in urosepsis syndrome: a prospective observational study. Crit Care. 2010;14(6):R206.

13. Park JH, Wee JH, Choi SP, Park KN. Serum procalcitonin level for the prediction of severity in women with acute pyelonephritis in the ED: value of procalcitonin in acute pyelonephritis. Am J Emerg Med. 2013;31(7):1092-7.

14. Ha YE, Kang Cl, Wi YM, Chung DR, Kang ES, Lee NY, Song JH, Peck KR. Diagnostic usefulness of procalcitonin as a marker of bacteremia in patients with acute pyelonephritis. Scand J Clin Lab Invest. 2013;73(5):444-8.

15. Schuetz P, Wirz Y, Sager R, Christ-Crain M, Stolz D, Tamm M, Bouadma L, Luyt CE, Wolff M, Chastre J, et al. Procalcitonin to initiate or discontinue antibiotics in acute respiratory tract infections. Cochrane Database Syst Rev. 2017;10:CD007498.

16. Rhee C. Using Procalcitonin to guide antibiotic therapy. Open forum infectious diseases. 2017, 4(1):ofw249.

17. de Jong E, van Oers JA, Beishuizen A, Vos P, Vermeijden WJ, Haas LE, Loef BG, Dormans T, van Melsen GC, Kluiters YC, et al. Efficacy and safety of procalcitonin guidance in reducing the duration of antibiotic treatment in critically ill patients: a randomised, controlled, open-label trial. Lancet Infect Dis. 2016:16(7):819-27.

18. Drekonja DM, Rector TS, Cutting A, Johnson JR. Urinary tract infection in male veterans: treatment patterns and outcomes. JAMA Intern Med. 2013; 173(1):62-8

Ready to submit your research? Choose BMC and benefit from:

- fast, convenient online submission

- thorough peer review by experienced researchers in your field

- rapid publication on acceptance

- support for research data, including large and complex data types

- gold Open Access which fosters wider collaboration and increased citations

- maximum visibility for your research: over $100 \mathrm{M}$ website views per year

At $\mathrm{BMC}$, research is always in progress.

Learn more biomedcentral.com/submissions 